\title{
Reconstruction of an extensive midfacial defect using additive manufacturing techniques
}

Nelson Fernandes BDS, ${ }^{1 *}$ Jacobus van den Heever, BChD, MChD (Pros), ${ }^{1}$ Christiaan Hoogendijk, MBChB, BChD, MChD (Chir. MaxFac Med), FCMFOS (SA), ${ }^{2}$ Sarel Botha, MBChB, BChD, Dip Odont (oral surg), MBA, MChD (MFOS), FCMFOS (SA), ${ }^{2}$ Gerrie Booysen, M.Tech (Eng Mech), ${ }^{3}$ Johan Els, B.Med.Sc (Hons) ${ }^{3}$

${ }^{1}$ Department of Prosthodontics, School of Dentistry, Faculty of Health Sciences, University of Pretoria, South Africa.

${ }^{2}$ Department of Maxillofacial and Oral Surgery, School of Dentistry, Faculty of Health Sciences, University of Pretoria, South Africa.

${ }^{3}$ Centre of Rapid Prototyping and Manufacturing, Central University of Technology, Bloemfontein, South Africa.

Midfacial defect reconstruction via additive manufacturing

*Address for correspondence: Dr NA Fernandes, Department of Prosthodontics, PO Box 1266, Pretoria, 0001, South Africa.

Tel: +27123192681; Fax: +27123230561; e-mail: nelsondentist@gmail.com

Disclosure: Supported by Southern Implants, Irene, South Africa for surface enhancement and sterilization of the implant, The South African National Research Foundation for financial support in acquiring a 3-D printer (Objet Connex 350), Materialise, Belgium for software support. No conflict of interest declared. This work was presented as a poster presentation by Dr J Van Der Heever from May 15-17 2014 at the "Oncologic dentistry and maxillofacial prosthetics symposium, University of Texas, Houston, Texas, USA". 


\section{ABSTRACT}

Malignant peripheral nerve sheath tumours are extremely rare tumours arising in peripheral nerves. Only 17 cases involving the trigeminal nerve have ever been reported. These tumours have a very poor prognosis and very high rates of recurrence and metastases. Their recommended treatment involves complete tumour resection followed by radiation. This can be problematic in the head and neck region. We present a clinical case involving a 33 year old female patient presenting with a slow growing exophytic mass of the anterior maxilla. Incisional biopsy and subsequent histological examination revealed a diagnosis of a malignant peripheral nerve sheath tumour. Surgical resection involved a complete maxillectomy, rhinectomy, and resection of the upper lip and aspects of the left and right cheeks. Reconstruction of the subsequent defect incorporated the placement of 4 zygomatic oncology implants to aid in retention of a facial prosthesis. These implants, however, were subsequently lost so it was decided to manufacture an anatomical model of the hard tissues via $3 \mathrm{D}$ printing. This model was used to design and manufacture a titanium frame (customised implant) for the patient, which was then fixated and secured intra-operatively with 21 cortical screws. A maxillary denture and silicone facial prosthesis were also made to fit onto this frame. This is the first known case where additive manufacturing, via the use of rapid prototyping and 3D printing, was employed to manufacture a facial prosthesis.

Keywords: rapid prototyping, 3D printing, facial prosthesis, malignant peripheral nerve sheath tumours.

\section{INTRODUCTION}

Malignant peripheral nerve sheath tumours (MPNST's) are extremely rare tumours arising from the Schwann cells of peripheral nerves. ${ }^{1}$ MPNST's of the trigeminal nerve are 
exceedingly rare $^{1}$ with only 17 cases being published in the English medical literature. ${ }^{2,5,6}$ These tumours have a very poor prognosis ${ }^{1}$ with 5 year survival rates of only $20 \%$ being reported in the head and neck region. ${ }^{1,8}$ Local recurrence rates range from $30-60 \%$ even in cases of complete tumour resection. ${ }^{1,3,7}$ They have been shown to mestastasize to the lungs, soft tissue, bone, liver, and/or brain in almost $65 \%$ of cases. ${ }^{1,2,3,10}$

Due to their aggressive and highly infiltrative nature ${ }^{1}$, high recurrence rates ${ }^{1,3}$, and poor prognosis, the recommended treatment for these tumours is aggressive radical resection. ${ }^{1,2,5,11}$ Complete removal of the lesion with tumour-free margins $\mathrm{s}^{3,4,9,10}$ measuring $2 \mathrm{~cm}$ in all directions is seldom possible in the head and neck region, therefore radiation therapy following resection is recommended. This could offer increased rates of tumour control and long-term survival. ${ }^{1,2,8,9,11}$

\section{CLINICAL REPORT}

We present the case of a 33 year old female patient who presented in 2011 with a slow growing exophytic soft tissue mass of the anterior maxilla in the region of the teeth 13,12 , $11,21, \& 22$ and spreading to the palate. A biopsy of the lesion was performed and histologically diagnosed as Kaposi sarcoma. The patient was referred for chemotherapy but, however, never received this treatment and no follow up appointments were scheduled.

The patient returned a few months later in 2012 presenting with an anterior palatal mass measuring 12x10x8mm which was infiltrating the underlying maxilla. The lesion appeared to be secondarily infected with signs and symptoms of continuous bleeding and occasional pain. An incisional biopsy was submitted for histological evaluation, which revealed a diagnosis of a malignant peripheral nerve sheath tumour. This required that the anterior portion of the 
maxilla be surgically resected, including all the anterior teeth, premolars, and the body of the maxilla up to $2 \mathrm{~cm}$ above the nasal floor.

The patient was once again non-compliant, so surgery and prosthesis fabrication was not performed at this time. She however did return again in 2013, presenting with tumour infiltration involving the left and right alae, the right cheek, as well as an ulcerated lesion of the upper lip, causing considerable pain and discomfort. An incisional biopsy and subsequent histological examination revealed a diagnosis of a recurrent malignant peripheral nerve sheath tumour (Figure 1).

Figure 1: Malignant peripheral nerve sheath tumour of the face

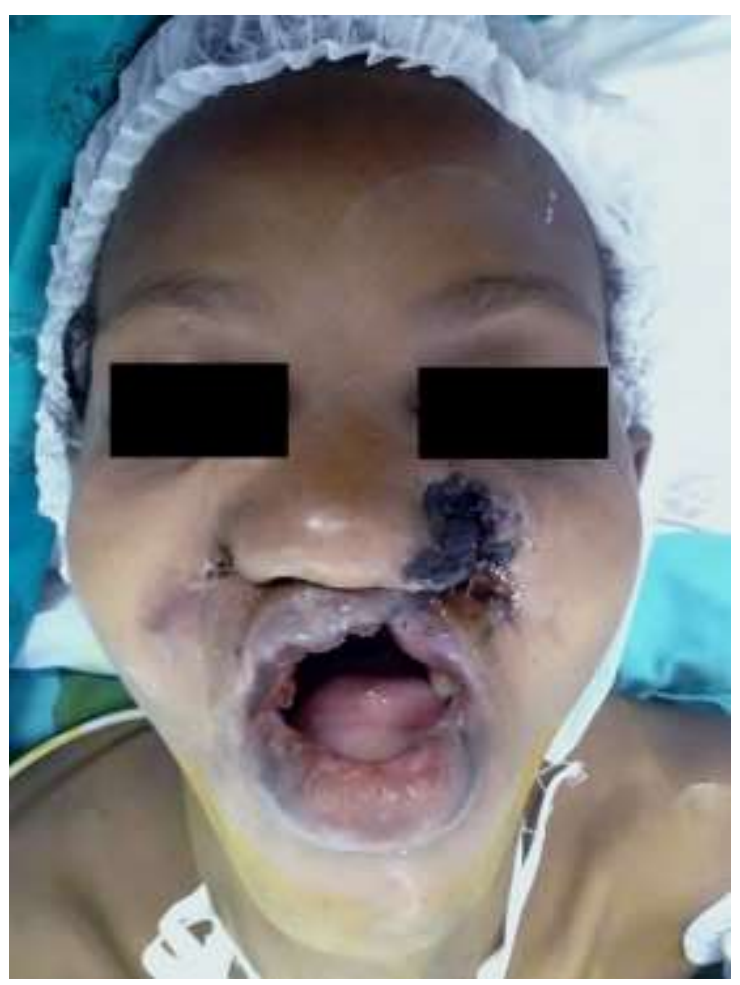

This tumour was extending rapidly, and due to a history of non-compliance by the patient, we had to act quickly. Our team, comprising two maxillo-facial surgeons and a prosthodontist, planned for the surgical excision of the tumour in its entirety. 


\section{Maxillectomy defects}

Maxillectomy defects resulting from resective surgery can be divided into the following types $^{12}$ :

- Type I- limited maxillectomy defects. These are partial defects where the palate and orbital floor remain intact.

- Type II- subtotal maxillectomy defects. These involve resection of most of the maxilla, but the orbital floor remains intact. In type IIA, the defect incorporates less than $50 \%$ of the palate, while in type IIB, the defect extends greater than $50 \%$ of the palate.

- Type III- total maxillectomy defects. This involves resecting the entire maxilla, which can exclude (Type IIIA) or include (Type IIIB) the orbital contents.

- Type IV- orbitomaxillectomy defects. This involves resection of the upper part of the maxilla and orbital contents with the palate remaining largely intact.

Due to its extent, a subtotal maxillectomy, rhinectomy, and resection of the upper lip including some aspects of the left and right cheeks would have to be performed. This placed our patient in the Type IIB category, as the resultant defect included the entire palate but the orbital floor and its contents remained in place.

Reconstruction of such a defect should ideally be done immediately or very soon after the resective procedure, so as to minimise psychological and emotional distress on the patient. The considerable gains in microvascular surgical techniques in the past 20-30 years have allowed surgeons to be able to reconstruct significant defects such as these in a single procedure. The use of pedicle flaps from the surrounding regions and free tissue transfers, including hard and soft tissues, from sites such as the radial forearm, rectus abdominus, 
fibula, scapular region and the iliac crest, have given surgeons the ability to customise their reconstructions to match defects. ${ }^{13}$

Surgical reconstruction of such a significant defect would have required an additional team of reconstructive and microvascular surgeons. Unfortunately, the public health service in our country lacks these highly specialized surgeons. It was thus decided to reconstruct the defect via an extensive maxillo-facial prosthodontic rehabilitation. No prior facial impressions were made before surgery due to a lack of available chair time in our prosthodontic ward.

Following the resective surgery, our maxillo-facial surgeons placed 4 zygomatic oncology implants (Southern Implants, South Africa). This was planned to aid in the retention of our prosthesis. At the same time, and still in the operating theatre, our prosthodontist took an impression of the defect and the position of the implants (Figure 2).

Fig. 2: Oncology implants placed at time of resection.

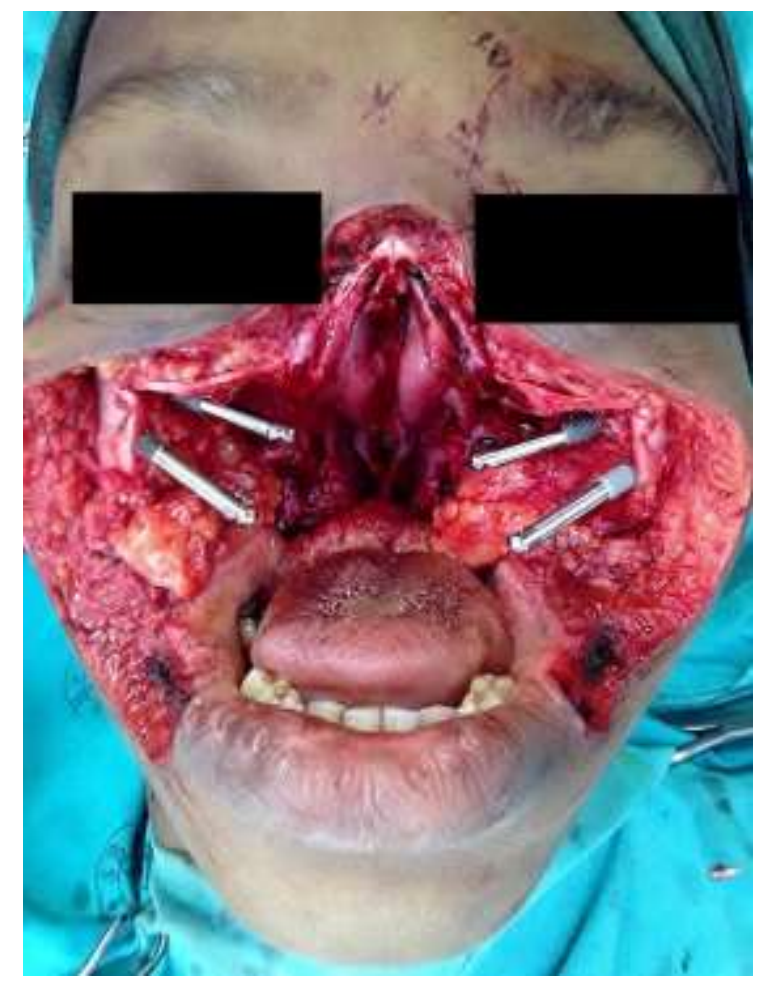

Zygomatic implants are $35-55 \mathrm{~mm}$ in length and are inserted into the zygomatic bone. As a result of this increased length, any movements on the implants will have significant 
leveraging effects at their point of insertion, which could interfere with osseointegration. At our institution, we try to splint these implants as quickly as possible. A modified provisional implant-supported obturator prosthesis and provisional silicone facial prosthesis were manufactured and placed three days following surgery. This was also done in order to establish separate oral and nasal cavities to improve swallowing, facilitate saliva control, and enhance speech articulation (Figures $3 \& 4$ ).

Fig. 3: Provisional implant supported acrylic obturator.

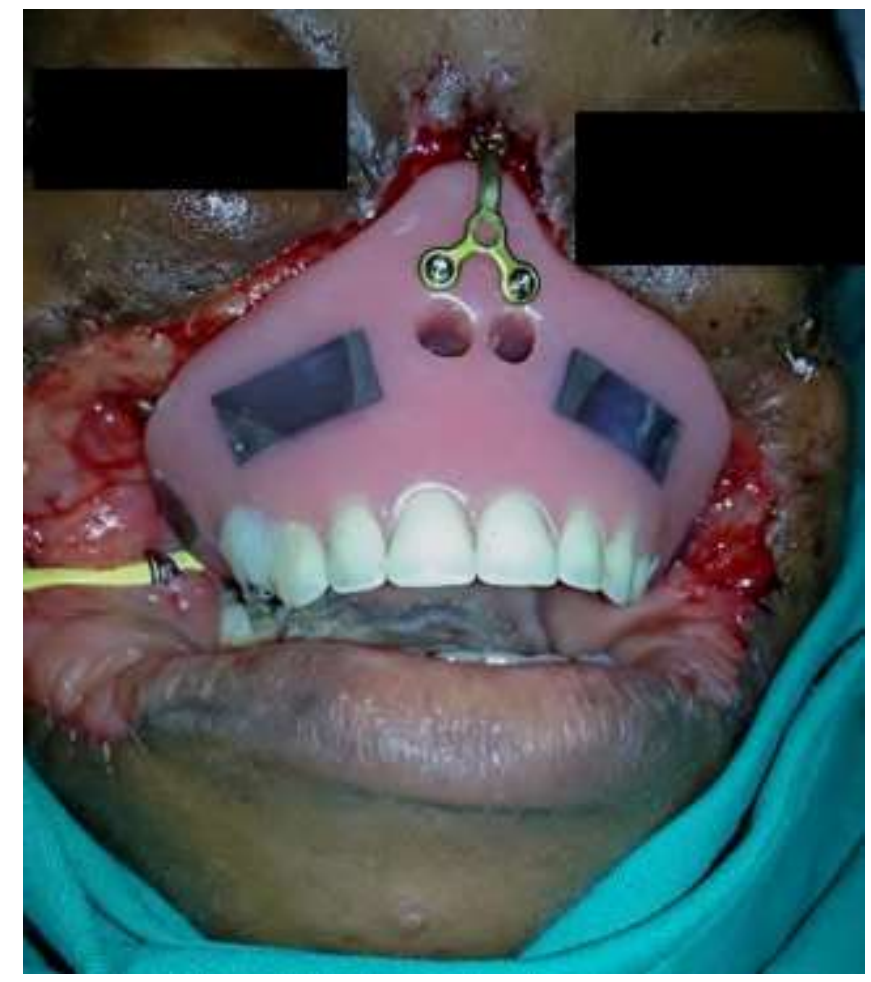


Fig. 4: Provisional facial prosthesis.

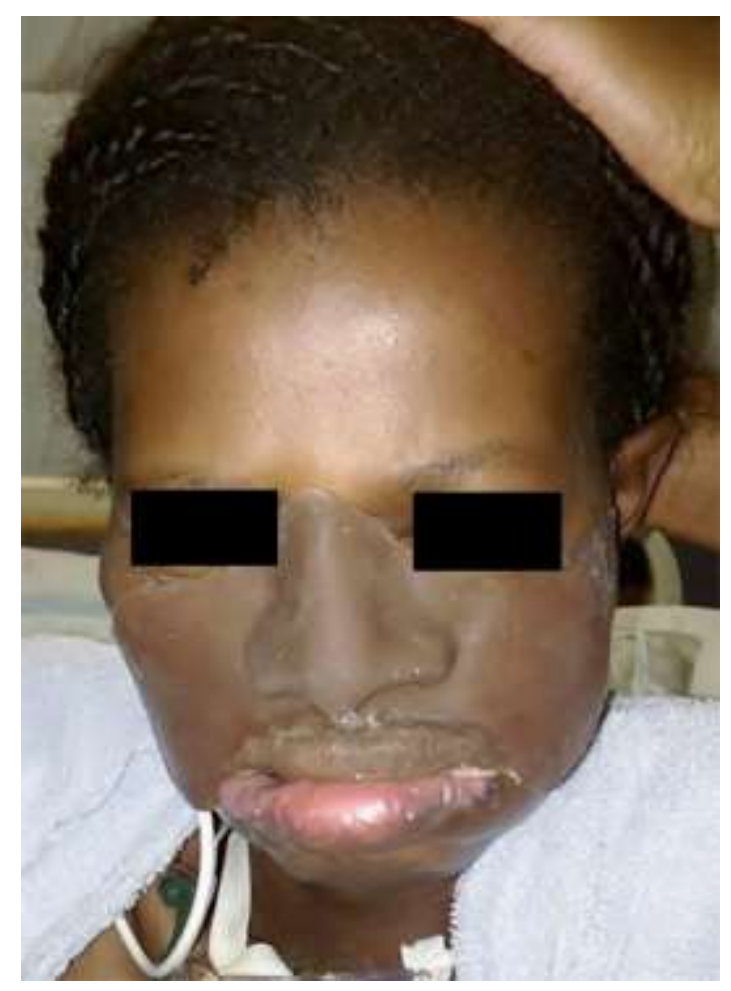

Fig. 5: Defect after loss of implants.

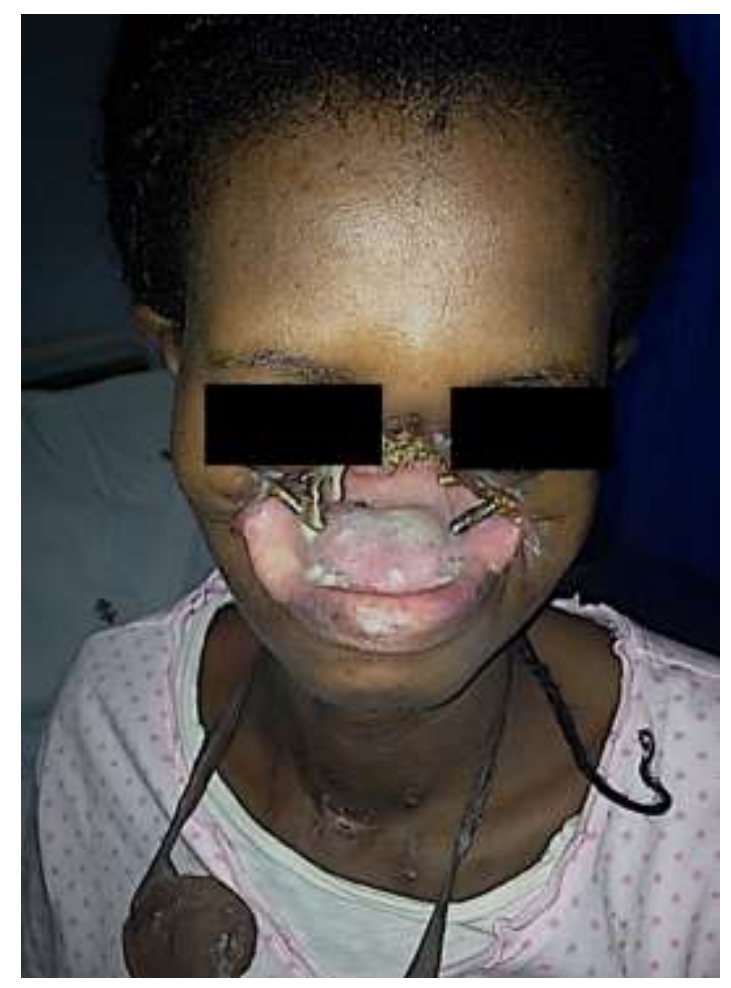


As a possible result of immediate loading on these implants, and the extensive size of the prosthesis loaded onto them, they failed to osseointegrate and were lost after 4 weeks. This meant that the obturator now had to be removed (Figure 5). This posed a major complication for the fabrication of a new prosthesis. The tissues bordering the defect had no bony foundation, and were easily compressed and distorted, which made subsequent impression taking virtually impossible. Traditional and conventional means for reconstruction were no longer an option, so we decided to incorporate advanced technology to assist us.

\section{Rapid prototyping and 3D printing}

As the name implies, rapid prototyping (RP) refers to the rapid manufacture of complex 3D models or physical parts (prototypes) using 3D computer aided design (CAD) data. It is widely used in product design in some industries and has found a use in the medical and dental fields over the past 30 years. In the dental field it is used specifically for improved and cost effective diagnosis, treatment planning, and manufacture of certain types of prostheses. ${ }^{14}$ Subtractive and additive fabrication methods can be used to manufacture physical prototypes. Whereas subtractive manufacturing is done via numerically controlled machining similar to a milling process to manufacture small objects, additive manufacturing is based on the decomposition of 3D computer model data into thin cross-sectional layers which is followed by physical formation and stacking of these layers into 3D forms. ${ }^{15}$

The development of computer aided design and computer aided manufacturing (CAD/CAM) relies on data acquisition, data processing, and manufacturing. The data in our case was obtained from computer tomography (CT) scans of our patient at $1 \mathrm{~mm}$ slice intervals (resolution). The data from the CT scanner was available as DICOM (digital imaging and communications in medicine) files which have to be converted into .stl (standard triangulation language) files, to then be used to input into our RP system. In order for us to do 
this, we utilized a computer programme known as MIMICS (Materialise interactive medical image control system, Materialise, Belgium), which was able to segment the image and provide a clearer representation of our area of interest. This software also allows one to alter the grey scale values from the DICOM images so as to differentiate between soft tissue and bone.

The segmentation of the CT scans was performed by engineers at the Centre for Rapid Prototyping and Manufacturing (CRPM, Bloemfontein, South Africa), where a team of engineers produced our 3D model on a laser sintering machine (P385, EOS, Germany). Selective laser sintering creates 3D models by fusing powdered materials with a carbon dioxide $\left(\mathrm{CO}_{2}\right)$ laser. The powdered material, available to us at a thickness of $150 \mu \mathrm{m}$, was a polyamide material known as PA2200 (EOS, Germany). This is a nylon based material which has been specifically formulated to meet FDA requirements and is thus suitable for medical use. This powdered material is spread by a roller over the surface of a build cylinder. A piston in the cylinder moves down one object layer thickness at a time to accommodate a new layer of powder. A laser beam traces over the surface of tightly compacted powder, thus elevating the temperature of the powder to its melting point. This then fuses the particles together and once all the layers are fused together it forms a solid mass, which in our case was our 3D model (Figure 6). 
Fig. 6: Frontal view of $3 \mathrm{D}$ planning model indicating the severity of the defect.

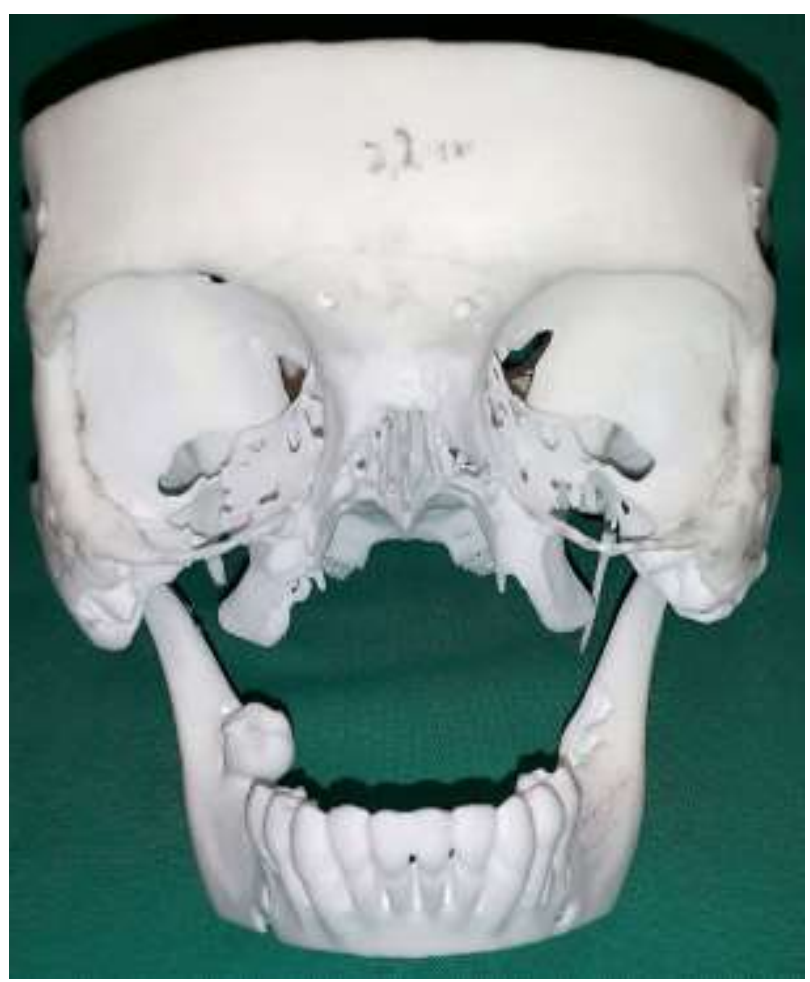

This model was essential to our planning as we were able to identify the boundaries and fixation areas of our planned titanium frame (implant). The 3D skull data from our CT scans was then imported into another software programme, namely 3-MATIC® (Materialise, Belgium). This software programme is truly unique in that it is capable of combining CAD tools with pre-processing (meshing) capabilities. It allows one to conduct thorough 3D measurements and analyses, and design implants and surgical guides specific to each patient. This allowed us to design the implant to be manufactured for our patient (Figure 7). 
Fig. 7: Final 3D CAD design of titanium implant.

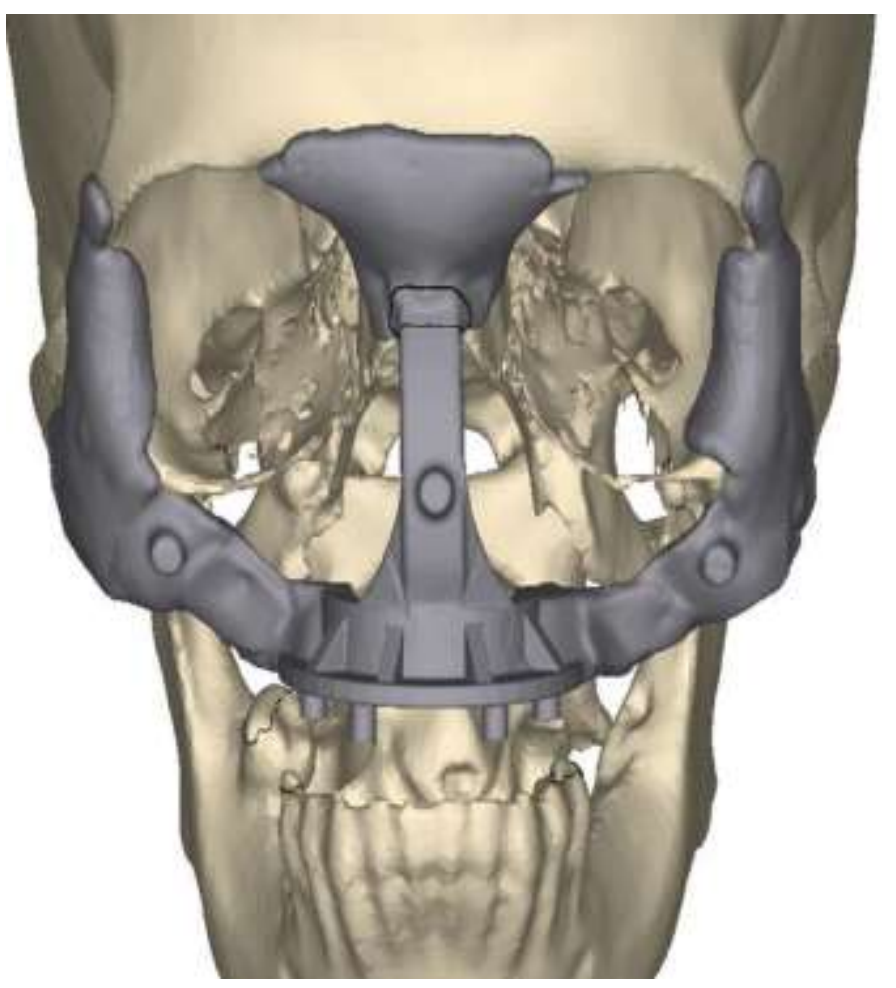

This design was printed in resin on an Objet Connex 350 machine (Stratasys, Germany) and positioned onto our original 3D model to ensure a precision fit. With our 3-MATIC® (Materialise, Belgium) software we were also able to design a custom drill guide for our surgeons, so that our fixation holes were placed at the correct angulation and location of where most suitable bone was located. In this process, we arrived at a total of 21 fixating holes which would anchor our implant with corresponding 21 cortical screws (Figure 8). 
Fig. 8: 3D design of drill guide with drilling angles indicated by the red lines.
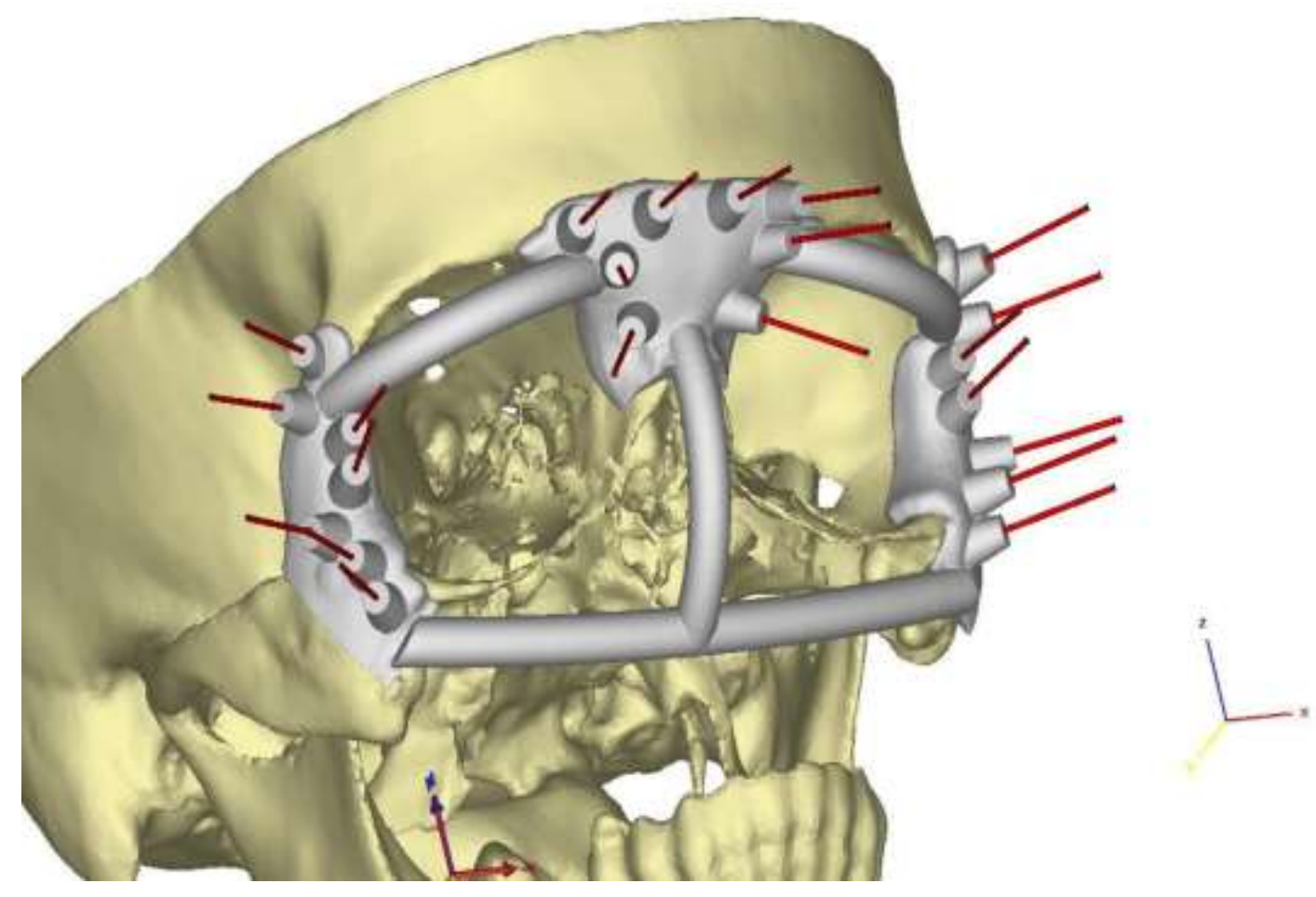

Our implant design was transferred as an .stl file to a direct metal laser sintering machine (EOSINT M280, EOS, Germany), which was able to manufacture our solid implant via a similar laser sintering process out of biocompatible titanium powder (titanium-6Al-4V) of particle size $<40 \mu \mathrm{m}$ (Figure 9).

This implant was trimmed and polished, after which the fitting surfaces of the implant were treated in much the same way as dental implants, which included surface abrasion. This was done so as to achieve possible cortical osseointegration. This procedure involved post machining and thread cutting carried out by Southern Implants (Irene, South Africa). The entire process from design to manufacture took 10 working days, with the cost amounting to around $\$ 5000$, for which we obtained sponsorship from our national research foundation, CRPM, and Materialise (Belgium). 
Fig. 9: Polished midfacial titanium implant.

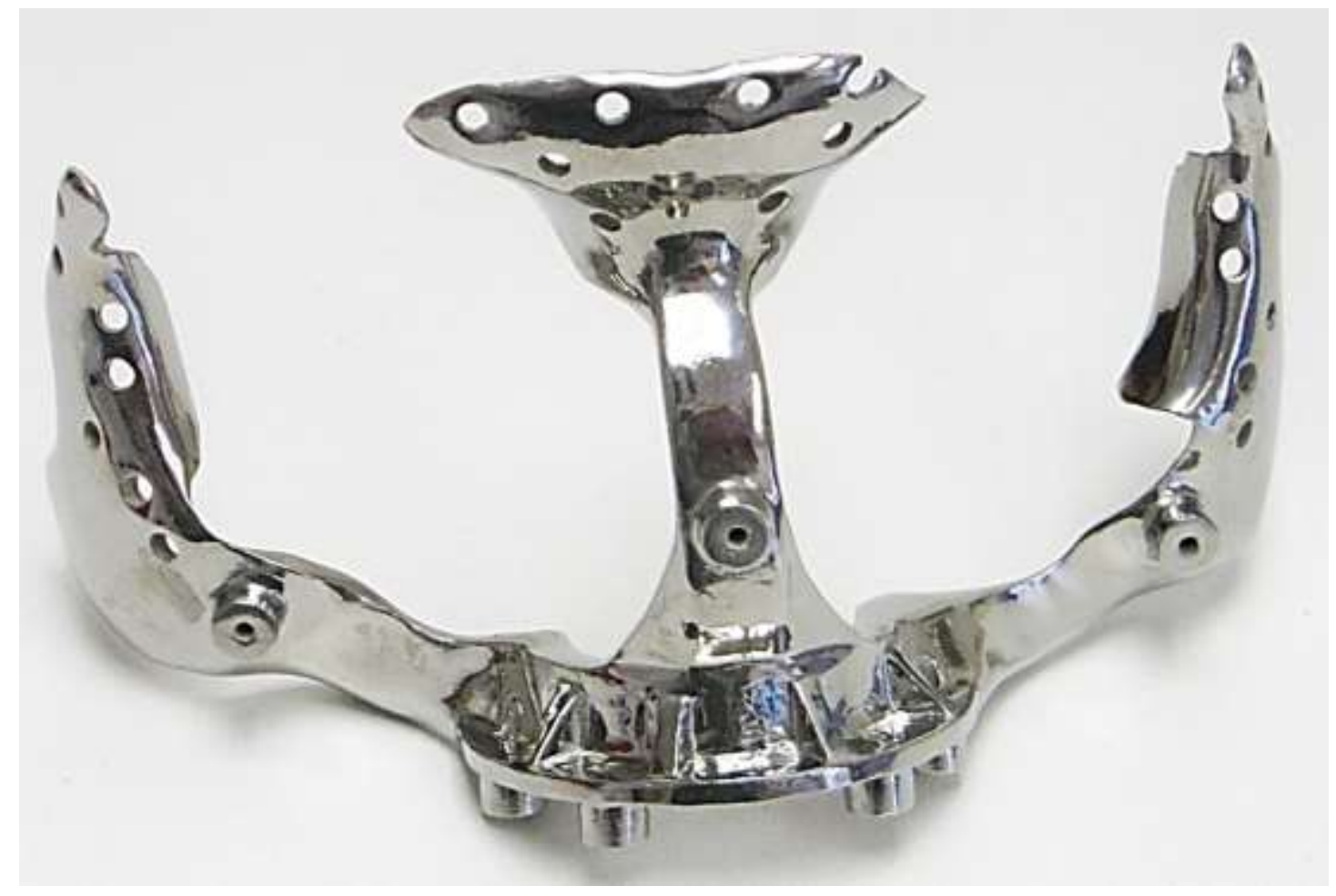

Fig. 10: Implant immediately post-surgery.

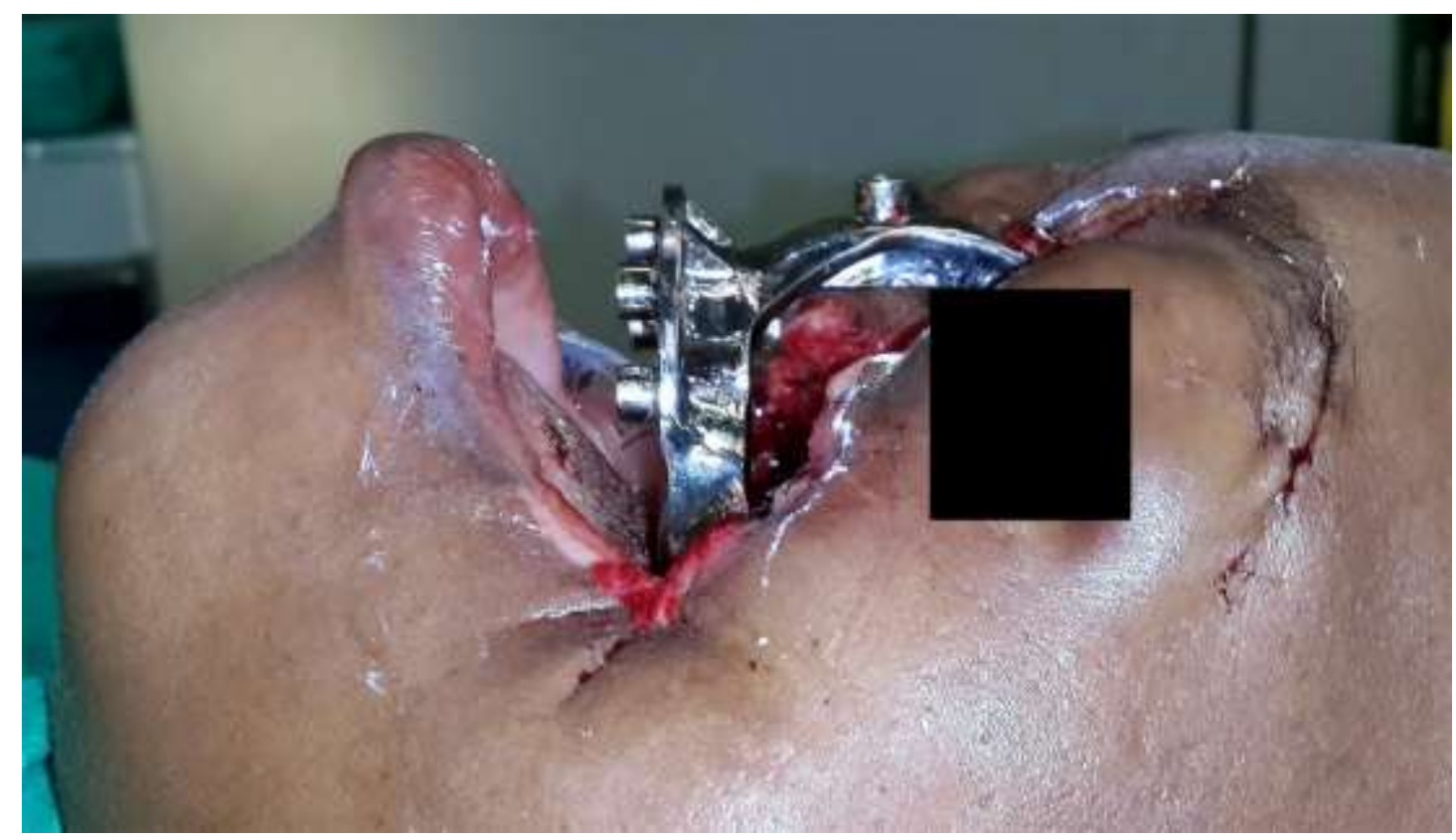

Our facial implant was placed intra-operatively and fixated as per our design with 21 cortical screws (Figure 10). A poly-vinyl-siloxane impression (President, Whaledent, USA) was also taken at this time with the implant in place for the manufacturing of a maxillary denture and a 
Fig. 11: Maxillary denture with soft base fitting surface.

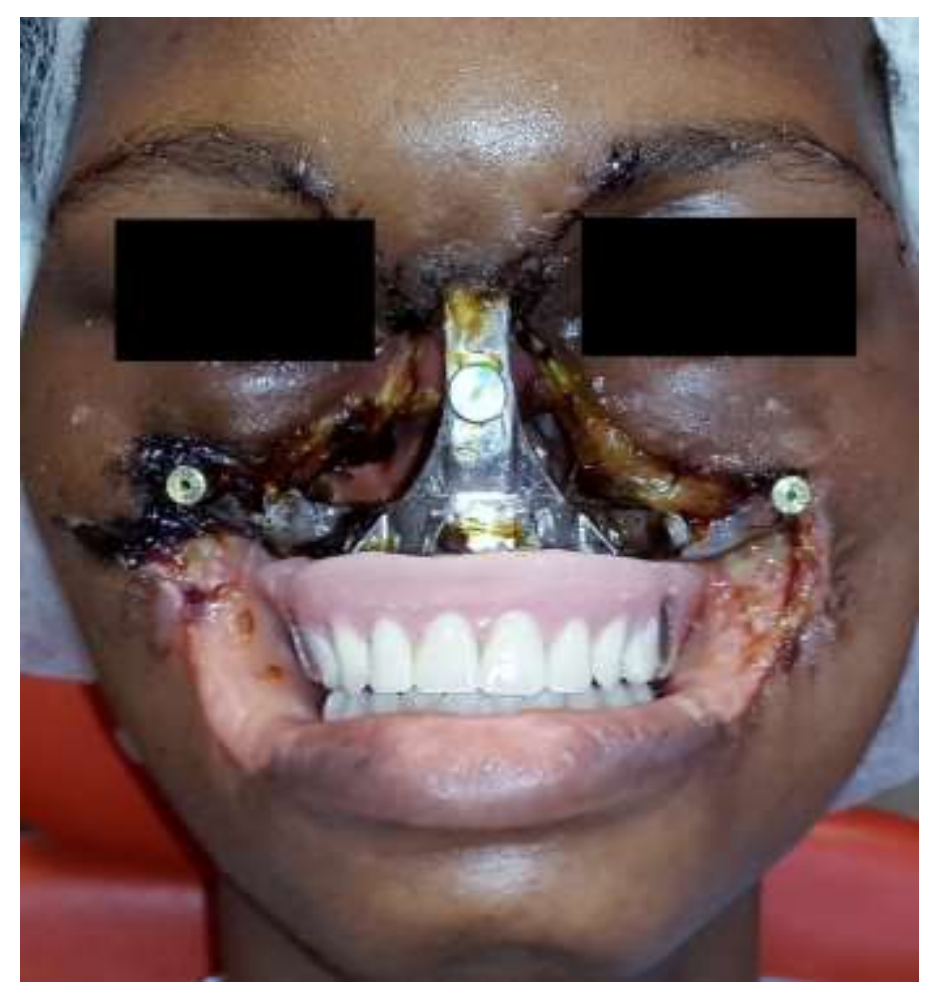

Fig. 12: Silicone facial prosthesis.

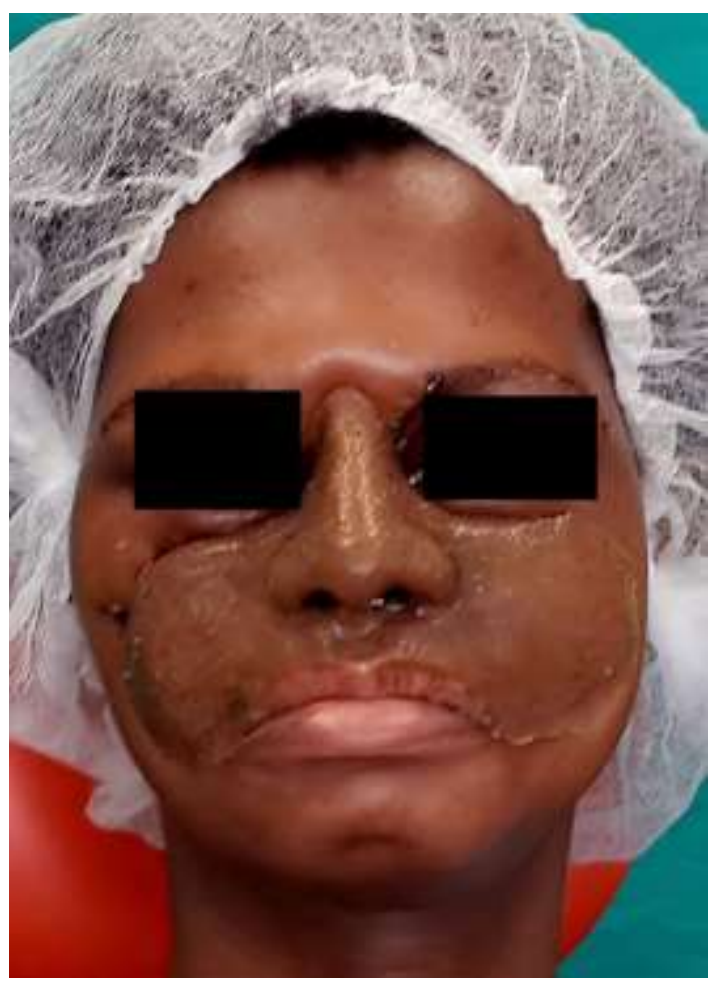


magnet-retained silicone facial prosthesis. Following 3 days, the finished maxillary denture's fitting surface was fitted and lined with a soft denture base material (Moloplast-B, USA) to act as a shock absorber during function (Figure 11), and our final facial prosthesis was delivered (Figure 12).

\section{CONCLUSION}

MPNST's of the trigeminal nerve are exceedingly rare. To our knowledge this is the first case where additive manufacturing techniques were used in manufacturing a custom titanium implant for the reconstruction of an extensive midfacial defect. Our patient has subsequently adapted well to her facial implant and prosthesis, and has been free of recurrent tumour for 6 months.

\section{REFERENCES}

1. Schmidt RF, Yick F, Boghani Z, et al: Malignant peripheral nerve sheath tumours of the trigeminal nerve: a systematic review of 36 cases. Neurosurg Focus 2013;34:1-10.

2. Bowers CA, Taussky P, Duhon BS, et al: Malignant peripheral nerve sheath tumour of the trigeminal nerve: a case report and literature review. Br J Neurosurg 2011;25:750-753.

3. Gupta G, Mammis A, Maniker A: Malignant peripheral nerve sheath tumours. Neurosurg Clin N Am 2008;19:533-543.

4. Hajdu SI: Peripheral nerve sheath tumours. Histogenesis, classification, and prognosis. Cancer 1993;72:3549-3552.

5. Chibbaro S, Herman P, Povlika M, et al: Malignant trigeminal scwannoma extending into the anterior skull base. Acta Neurochir (Wien) 2008;150:599-604. 
6. Sheitnauer BW, Erdogan S, Rodriguez FJ, et al: Malignant peripheral nerve sheath tumours of cranial nerves and intracranial contents: a clinicopathologic study of 17 cases. Am J Surg Pathol $2009 ; 33: 325-338$

7. MacNally SP, Rutherford SA, Ramsden RT, et al: Trigeminal schwannomas. Br J Neurosurg 2008;22:729-738.

8. Minovi A, Basden O, Hunter B, et al: Malignant peripheral nerve sheath tumours of the head and neck: management of 10 cases and literature review. Head Neck 2007;29:439-445.

9. Ducatman BS, Scheithauer BW, Piepgras DG, et al: Malignant peripheral nerve sheath tumours. A clinicopathologic study of 120 cases. Cancer 1986;57:2006-2021.

10. Stark AM, Buhl O, Hugo HH, et al: Malignant peripheral nerve sheath tumours- report of 8 cases and review of the literature. Acta Neurochir (Wien) 2001;143:357-364.

11. Ziadi A, Saliba I: Malignant peripheral nerve sheath tumour of intracranial nerve: a case series review. Auris Nasus Larynx 2010;37:539-545.

12. Cordeiro PG, Chen CM: A 15-year review of midface reconstruction after total and subtotal maxillectomy: Part I. Algorithm and outcomes. Plast Reconstr Surg 129: 129-136.

13. Futran ND, Mendez E: Developments in reconstruction of midface and maxilla. Lancet Oncol 2006;7:249-258.

14. Sun J, Zhang F: The application of rapid prototyping in prosthodontics. J Prosthodont 2012;21:641-644.

15. Azari A, Nikzad S: The evolution of rapid prototyping in dentistry: a review. Rapid prototyping journal 2009;15:216-225. 